\title{
ATYPICAL PRESENTATION OF SYSTEMIC LUPUS ERYTHEMATOSUS IN A WEST AFRICAN MALE
}

\author{
KWAME YEBOAH-MENSAH \\ Rheumatology Clinic, Directorate of Medicine, Komfo Anokye Teaching Hospital, Kumasi, Ghana
}

DOI: http://dx.doi.org/10.4314/gmj.v48i1.9

Corresponding Author: Dr. Kwame Yeboah-Mensah

Email: dryeboahmensah@live.com

Conflict of Interest: None declared

\section{SUMMARY}

Systemic lupus erythematosus (SLE) is a chronic, autoimmune multi-system disorder. About seventy to ninety percent of all cases of SLE occur in women. Although the disease is common in black young women residing in Europe and North America, it is reputed to be a very rare diagnosis in West Africa. A case of atypical presentation of SLE in a male in West Africa is presented.

Keywords: SLE, male. aphthous ulcers, paraparesis, anti-Smith antibodies

\section{INTRODUCTION}

Systemic lupus erythematosus (SLE) is a chronic autoimmune inflammatory disease which can present with different combinations of symptoms and also tends to mimic other medical conditions, thus making it difficult to diagnose without delay in most cases. The disease is more prevalent in the age group 15 to 44 years and occurs $90 \%$ of the time in females. ${ }^{1}$ The disease is said to be rare in West Africa in earlier publications. ${ }^{2,3}$

The low rate of documented diagnosis in West Africa may result from low index of suspicion of the diagnosis in patients presenting with atypical features of the disease and also inadequate laboratory support for the diagnosis or a low level of reporting. Studies show that SLE tends to occur at an early age in males as compared to females. ${ }^{4}$ The classic triad of fever, rash and joint pains, which was described in earlier literature ${ }^{5}$ as characterizing most cases of SLE does not always occur in the initial presentation which may further delay the diagnosis.

Generally, males tend to have increased frequency of discoid lupus, thrombocytopenia, neuropsychiatric and renal involvement at time of diagnosis. ${ }^{6}$
A case of SLE with atypical presentation in a 52-year old male in West Africa is presented here to highlight the need for a high index of suspicion for such cases.

\section{CASE REPORT}

A 52-year old Ghanaian male was admitted to the with a two-month history of worsening generalized malaise and weight loss. Patient had anorexia, but denied nausea, vomiting, fever, dysuria, cough, diarrhoea or skin rash. He complained of hesitancy and straining with micturition for about two weeks, and had experienced a single episode of gross haematuria about twenty four hours prior to presentation to the hospital. He had passed very scanty non-bloody urine on the day of admission.

His past medical history was remarkable only for hypertension diagnosed about 4 years earlier, and for which he took $30 \mathrm{mg}$ orally of sustained-release nifedipine daily. He denied any known drug allergies, and denied tobacco or alcohol use.

The initial vital signs were normal. There was mild pallor with no icterus or lymphadenopathy. There were discrete non-tender ulcers on the roof of the mouth and on the buccal mucosa. The cardiovascular and respiratory examination findings were unremarkable. The abdominal examination showed a tender distended bladder, but no hepato-splenomegaly. Patient was conscious and alert with normal speech. Muscle power was grade 5 in all limbs and Kernig sign was absent.

A Foley catheter was inserted to relieve the acute urinary retention. At about 18 hours after admission, patient was noted to have rapid onset flaccid paraparesis with muscle strength of $1 / 5$ in both lower extremities, but with no sensory deficits to pain or light touch. 
His laboratory test results are as follows: Total white cell count on two occasions was less than $4 \times 10^{9} / \mathrm{L}$ (leucopoenia). Haemoglobin was 9.6 then $10.3 \mathrm{~g} / \mathrm{dl}$ whilst platelets were normal. Serum folate, B12 and CRP were normal. Serum iron and transferrin were low and ESR persistently above $100 \mathrm{~mm} / \mathrm{hr}$.

Blood urea and electrolytes were normal, urinalysis showed increased red and white cells, proteinuria and white cell casts. Repeat urinalysis was normal.

Other urinary tests results showed 24-hour urinary protein of $0.44 \mathrm{~g} / 24$ hours, no malignant cells on cytology, negative PCR for tuberculosis and no bacterial growth on culture.

Other blood tests performed, which were normal, include prostate specific antigen, HIV antibody tests, hepatitis B surface antigen, rheumatoid factor, AntiCyclic Citrullinated peptide, C-ANCA, P-ANCA and complement 3 and 4. Anti IFA titres, anti-ds-DNA, anti Ro and anti-La were all negative. The ANF pattern was speckled, Anti -Sm Ab and Anti-RNP68 Ab positive.

A stool occult blood test was negative and serum protein electrophoresis showed polyclonal gammopathy. A chest $\mathrm{x}$-ray and, plain $\mathrm{x}$-ray of lumbar sacral spine were normal. Abdominal/Pelvic ultrasound showed a large (10.6 x $7.5 \mathrm{~cm} 2)$ simple cyst occupying the upper pole of the left kidney. The prostate was homogenously enlarged with a volume of about 45.1 millilitres.

CT scan of abdomen/pelvis showed enlarged prostate with mild bilateral hydronephrosis. The liver, spleen, pancreas and adrenal glands appeared normal with no ascites or retroperitoneal lymphadenopathy. MRI of spine was not done due to unavailability. Cystoscopy showed no bladder tumour with normal ureteric orifices. Prostate biopsy showed no evidence of malignancy. Renal Biopsy was not done.

A diagnosis of systemic lupus erythematosus was made after excluding other diagnosis based on the presenting features of aphthous ulcers, leucopoenia, and cellular casts in the urine, positive antinuclear and anti-Smith antibodies. Patient was started on high dose prednisolone which was tapered over two months to $10 \mathrm{mg}$ daily. He was also started concurrently on hydroxychloroquine $200 \mathrm{mg}$ daily. He participated in daily physiotherapy for four weeks.

The urinary retention was evaluated by the urology service for which he was prescribed tamsulosin and dutasteride. The Foley catheter was successfully removed after five weeks. He was also placed on Lisinopril and amlodipine for blood pressure control.
After one month on therapy, patient had improved remarkably. The strength in both lower extremities had returned to normal and the aphthous ulcers had disappeared. At follow up visit after two months on therapy, he had regained his baseline muscle power. All abnormal laboratory parameters had returned to normal.

\section{DISCUSSION}

The classification criteria for systemic lupus erythematosus were updated in 1997. At least four of these eleven criteria (in Table 1) are required to classify patients as having systemic lupus erythematosus.

Table 1 Criteria for the Classification of Systemic Lupus Erythematosus

At least 4 of the following are required to classify patients as having SLE in reports of clinical research:

\begin{tabular}{|l|}
\hline Malar rash \\
\hline Discoid rash \\
\hline Photosensitivity \\
\hline Oral ulcers \\
\hline Arthritis \\
\hline Serositis \\
\hline Renal disorder \\
\hline $\begin{array}{l}\text { Leukopenia }(<4000 / \mu \mathrm{L}), \text { lymphopenia }(<1500 / \mu \mathrm{L}), \\
\text { haemolytic anaemia, or thrombocytopenia } \\
100,000 / \mu \mathrm{L})\end{array}$ \\
\hline Neurologic disorder \\
\hline $\begin{array}{l}\text { Positive test for anti-DNA, anti-Smith, or antiphospho- } \\
\text { lipid antibodies }\end{array}$ \\
\hline Antinuclear antibodies in high titres \\
\hline
\end{tabular}

This patient's presentation fulfilled five of these eleven criteria for a definite diagnosis of systemic lupus erythematosus. Certain aspects of this patient's presenting features are quite atypical for classic SLE at initial presentation and could have delayed the diagnosis. This discussion will focus on the four atypical aspects of his presentation.

Ethnicity, age and gender: Several studies have demonstrated a marked ethnic/geographic variance in the prevalence and severity of SLE around the world. There is increased prevalence and severity in people of African-American and Afro-Caribbean backgrounds resident in North America or Europe, but SLE is rarely described in West Africa from where most migrant African-American and Afro-Caribbean populations originated. $^{3}$

There are no population-based studies of SLE in West Africa, and by 2009 , there were about thirty three case reports from the West African sub-region. 
This could be attributable to low level of reporting of cases of SLE. On the other hand the study by Molokhia et al suggested that the disease is not rare in West Africa. ${ }^{7}$

SLE has long been regarded as a disease of young women in their twenties and thirties. Maddock Jr. et al noted that men develop SLE less frequently with no apparent concentration in any age group. ${ }^{8}$ Studies have shown that young women present classically with c fever, joint pains and rash $^{9}$, whereas men tend to have increased frequency of discoid lupus, thrombocytopaenia, neuropsychiatric and renal involvement at the time of diagnosis. ${ }^{7}$

Diagnosis of SLE requires a high index of suspicion. The combination of aphthous ulcers, high erythrocyte sedimentation rate, and weight loss, anaemia of chronic disease, polyclonal gammopathy, leukopenia, proteinuria and white blood cell cast in the absence of obvious infection or malignancy raised suspicion for chronic autoimmune disease, especially SLE despite the atypical presentation, sex and age. SLE was subsequently confirmed by increased antinuclear antibody and anti-Smith antibody.

Complements $\mathrm{C} 3$ and $\mathrm{C} 4$ are acute phase reactants that may manifest increased serum levels in serum during inflammatory flares of SLE. On the other hand, increased consumption during flares may result in hypocomplementaemia. Harvey et al noted in their review of SLE that low serum complement C4 is very infrequent in over the age forty years. ${ }^{10}$ The patients presentation was thus atypical.

According to the American College of Rheumatology, a total of 19 neuropsychiatric syndromes that can occur in the course of SLE have been described and defined.

The most common syndromes are cognitive disorders, mood disorders, headaches, seizures and cerebrovascular disease. Myelopathy is one of the less common neuropsychiatric manifestations of systemic lupus erythematosus with a reported prevalence of about $1-3 \%$. $^{11}$

Transverse myelitis commonly presents as a late manifestation of systemic lupus erythematosus, but there have been reports of transverse myelitis presenting at disease onset with high association with antiphospholipid antibodies and with good response to treatment. ${ }^{12}$

This patient's paraparesis which was presumed to be secondary to myelopathy could not be evaluated further with antiphospholipid antibody tests, MRI, myelogram or nerve conduction velocities due to limited resources.
Nonetheless, this patient made a rapid and complete recovery of motor function in both lower extremities following initiation of steroids, hydroxychloroquine and physiotherapy.

Presence of anti-Smith and Anti-RNP antibodies: Antibodies to double-stranded DNA are often measured in SLE and are useful in the diagnosis of the condition, but the levels tend to fluctuate and they may be absent at times as was observed in this patient. Antibodies directed against small nuclear riboprotein include antiSmith antibody and antiribonuclear protein (anti-RNP) antibodies.

They bind to related but distinct antigens. Anti-Smith antibodies can be used for confirming the diagnosis of SLE. They have a low a sensitivity of $24 \%$ to $30 \%$ but a high specificity of $96 \%$ to $98 \%$. Anti-Smith antibodies are known to be more prevalent in black patients. ${ }^{13}$ Anti-RNP antibodies coexist with anti-Smith antibodies in many patients with systemic lupus erythematosus. ${ }^{14}$ It is therefore important for clinicians in West Africa to test for the anti-Smith and anti-RNP antibodies in addition to anti ds-DNA antibodies in patients suspected to have SLE.

\section{CONCLUSION}

Systemic lupus erythematosus is uncommon in males and atypical presentation may make the diagnosis extremely difficult. This case report seeks to draw medical practitioners' attention in the West African sub region to the probability of systemic lupus erythematosus in males as well as the possibility of presentation with very atypical features. Laboratory tests to aid diagnosis may be unaffordable and clinicians need to have a high index of suspicion to be able to recognise the disease in patients who present atypically.

\section{REFERENCES}

1. Chakravarty EF, Bush TM, Manzi S et al. Prevalence of adult systemic lupus erythematosus in California and Pennsylvania in 2000: estimates obtained using hospitalization data. Arthritis Rheum 2007; 56: 2092.

2. Fessel J. Systemic lupus erythematosus in the community: incidence, prevalence, outcome and first symptoms; the high prevalence in black women. Arch Intern Med 1974; 134: 1027-1035.

3. Greenwood BM. Autoimmune disease and parasitic infections in Nigerians. Lancet 1968; 11: 380382 .

4. Sthoeger ZM, Geltner D, Rider A, Bentwich Z. Systemic lupus erythematosus in 49 Israeli males:

5. retrospective study. Clin Exp Rheumatol 1987; 5: 233-40. 
6. Dubois EL, Tuffanelli DL. Clinical manifestations of systemic lupus erythematosus. Computer analysis of 520 cases. JAMA Oct 12 1964; 190: 104-11.

7. Molina JF, Drenkard C, Molina J, Cardiel MH, Uribe O, Anaya JM et al. Systemic lupus erythematosus in males. A study of 107 Latin American patients. Medicine (Baltimore) 1996; 75: 124-30.

8. Molokhia M, McKengue PM, Cuadrado M, Hughes G. Systemic lupus erythematosus in migrants from West Africa compared with afroCaribbean people in the UK. Lancet 2001 May 5; 357 (9266): 1414-5.

9. Maddock Jr RK. Incidence of systemic lupus erythematosus by age and sex. JAMA 1965 Jan 11; 191: 137-138.

10. Isah AO, Obasohan AO, Oyewo EA, OhajuObodo J. Amlodipine versus nifedipine in the treatment of mild-to-moderate hypertension in black Africans. Current Therapeutic Research Volume 57; Issue 4: 300-308.

11. Harvey AM, Schulman LE, Tumulty PA, Conley CL, Schoenrich EH. Systemic lupus erythemato
12. sus: review of the literature and clinical analysis of 138 cases. Medicine (Baltimore) Dec 1954; (4): 291-437.

13. Kovacs B, Lafferty TL, Brent LH, DeHoratius RJ. Transverse myelopathy in systemic lupus erythematosus: an analysis of 14 cases and review of the literature. Ann Rheum Dis 2000; 59: 120-4.

14. D'Cruz DP, Mellor-Pita S, Joven B, Sanna G, Allanson J, Taylor J, Khamashta MA, Hughes GRV. Transverse myelitis as a first manifestation of systemic lupus erythematosus or lupus-like disease: good functional outcome and relevance of antiphospholipid antibodies. J Rheumatol 2004 Feb; 31(2): 280-5.

15. Migliorini P, Baldini C, Rocchi V, Bombardieri S. Anti-Sm and anti-RNP antibodies. Autoimmunity 2005 Feb; 38(1): 47-54.

16. American College of Rheumatology Ad Hoc Committee on Immunologic Testing Guidelines. Guidelines for immunologic testing in the rheumatic diseases: Anti-Sm and anti-RNP antibody tests. Arthritis Rheum 2004; 18: 249-269. 